\title{
TMD parton shower effects in associated $\gamma+$ jet production at the LHC
}

\author{
A. V. Lipatov, ${ }^{1,2}$ M. A. Malyshev $\odot,{ }^{1}$ and H. Jung $\oplus^{3}$ \\ ${ }^{1}$ Skobeltsyn Institute of Nuclear Physics, Lomonosov Moscow State University, 119991 Moscow, Russia \\ ${ }^{2}$ Joint Institute for Nuclear Research, 141980 Dubna, Moscow Region, Russia \\ ${ }^{3}$ Deutsches Elektronen-Synchrotron, 22603 Hamburg, Germany
}

(Received 28 June 2019; published 29 August 2019)

\begin{abstract}
We investigate associated prompt photon and hadronic jet production at the LHC energies using the $k_{T^{-}}$ factorization approach. Our consideration is based on the $\mathcal{O}\left(\alpha \alpha_{s}^{2}\right)$ off-shell gluon-gluon fusion subprocess $g^{*} g^{*} \rightarrow \gamma q \bar{q}$ and several subleading quark-initiated contributions from $\mathcal{O}\left(\alpha \alpha_{s}\right)$ and $\mathcal{O}\left(\alpha \alpha_{s}^{2}\right)$ subprocesses, taken into account in the conventional (collinear) QCD factorization. The transverse momentumdependent (or unintegrated) gluon densities in a proton are derived from Catani-Ciafaloni-FioraniMarchesini evolution equation. We achieve reasonably good agreement with the experimental data taken by CMS and ATLAS Collaborations and demonstrate the importance of initial-state parton showers for jet determination in the $k_{T}$-factorization approach.
\end{abstract}

DOI: 10.1103/PhysRevD.100.034028

\section{INTRODUCTION}

Investigation of prompt photon and associated hadronic jet production is an important topic of modern experimental and theoretical research [1-6]. The photons are called prompt if they originate from the hard partonic subprocess, rather than from secondary decays. Such events provide a direct probe of the hard subprocess dynamics since the produced photons are largely insensitive to the effects of final-state hadronization. The measured $\gamma+$ jet total and differential cross sections are sensitive to the quark and gluon densities in the proton over the whole kinematical region of longitudinal momentum fraction $x$ and hard scale $\mu^{2}$ and represent an important background to many processes involving photons in the final state, including Higgs boson production (in the diphoton decay mode). Thus, it is essential to have accurate QCD predictions for corresponding cross sections.

The reported measurements [1-6] are in agreement with the results of next-to-leading-order (NLO) perturbative QCD calculations performed using the JETPHOX Monte Carlo event generator [7]. The leading-order (LO) calculations based on the Monte Carlo event generator SHERPA [8], which incorporates higher-order tree-level matrix elements and parton shower modeling, also agree well with the measurements [1-6]. An alternative description of $\gamma+$ jet data can be achieved in the framework of the

Published by the American Physical Society under the terms of the Creative Commons Attribution 4.0 International license. Further distribution of this work must maintain attribution to the author(s) and the published article's title, journal citation, and DOI. Funded by SCOAP. high-energy QCD factorization [9], or $k_{T}$-factorization approach [10]. This approach is based on the BalitskyFadin-Kuraev-Lipatov (BFKL) [11] or Ciafaloni-CataniFiorani-Marchesini (CCFM) [12] gluon evolution equations and has certain technical advantages in the ease of including higher-order QCD radiative corrections [namely, part of $\mathrm{NLO}+$ next-to-next-to-leading-order $+\ldots$ terms corresponding to real initial-state gluon emissions] that can be taken into account in the form of transverse momentumdependent (TMD, or unintegrated) parton distributions. ${ }^{1}$ It has become a widely exploited tool and it is of interest and importance to test it in as many cases as possible.

In the present paper, we apply the $k_{T}$-factorization approach to the associated $\gamma+$ jet production at LHC energies, which continues the line of our previous studies [15-17] in which we have inspected inclusive photon as well as associated prompt photon (or rather $Z$ boson) and heavy quark jet production. Note that the associated $\gamma+$ jet production was already examined in the $k_{T}$-factorization framework $[18,19]$. In particular, some photon-jet correlations have been studied at the Relativistic Heavy Ion Collider and Tevatron energies [18]. However, initial-state parton showers, which are important for the proper jet determination in the $k_{T}$-factorization approach, have been not taken into account in those calculations. A simple model [20] to implement the effects of parton showers into analytical calculations results in some difficulties in simultaneous description of photon transverse momentum and rapidity distributions in the whole kinematical range [19] (see the discussion below in Sec. II). The importance of parton shower contributions to jet production was pointed

\footnotetext{
${ }^{1}$ See Refs. [13,14] for more information.
} 
out [21], and the method [22] to reconstruct correctly the kinematics of the jets with taking into account TMD parton showers was proposed. The major goal of the present article is to apply the method [22] to associated $\gamma+$ jet production and improve our previous results [19] using a TMD shower implemented in the Monte Carlo event generator CASCADE [23]. Our other goal is the selection of TMD gluon densities in a proton best suited to describe the available experimental data.

The outline of the paper is the following. In Sec. II, we briefly describe our approach. In Sec. III, we present the results of our calculations and confront them with the available data. Our conclusions are summarized in Sec. IV.

\section{THEORETICAL FRAMEWORK}

Let us briefly describe the calculation steps. We start from the off-shell gluon fusion subprocess,

$$
g^{*}\left(k_{1}\right)+g^{*}\left(k_{2}\right) \rightarrow \gamma(p)+q\left(p_{1}\right)+\bar{q}\left(p_{2}\right),
$$

where the momenta of all particles are given in parentheses. The corresponding gauge-invariant off-shell production amplitude was calculated earlier [24,25] and implemented into the Monte Carlo event generator CASCADE [23] and newly developed parton-level Monte Carlo event generator PEGASUS [26]. All the details of these calculations were explained in Refs. [24,25]. We only mention here that evaluation of the off-shell matrix element involves a special gluon polarization sum rule,

$$
\sum \epsilon^{\mu} \epsilon^{* \nu}=\frac{k_{T}^{\mu} k_{T}^{\nu}}{\mathbf{k}_{T}^{2}},
$$

where $\epsilon$ is the gluon polarization vector and $k_{T}$ its nonzero transverse momentum. In the collinear limit $\mathbf{k}_{T}^{2} \rightarrow 0$, this expression converges to the ordinary one after averaging over the azimuthal angle. In all other respects, the calculations follow the standard QCD Feynman rules.

Following Ref. [17], in addition to off-shell gluon-gluon fusion, we take into account several subprocesses involving quarks in the initial state, namely,

$$
\begin{gathered}
q\left(k_{1}\right)+g\left(k_{2}\right) \rightarrow \gamma(p)+q\left(p_{1}\right), \\
q\left(k_{1}\right)+\bar{q}\left(k_{2}\right) \rightarrow \gamma(p)+g\left(p_{1}\right), \\
q\left(k_{1}\right)+q^{\prime}\left(k_{2}\right) \rightarrow \gamma(p)+q\left(p_{1}\right)+q^{\prime}\left(p_{2}\right), \\
q\left(k_{1}\right)+\bar{q}\left(k_{2}\right) \rightarrow \gamma(p)+q^{\prime}\left(p_{1}\right)+\bar{q}^{\prime}\left(p_{2}\right),
\end{gathered}
$$

where the momenta of all particles are given in parentheses. Despite the fact that quark densities are typically much lower than the gluon density at LHC conditions, these processes may become important at very large transverse momenta (or at large parton longitudinal momentum fraction $x$, which is needed to produce large $p_{T}$ events) in which the quarks are less suppressed or can even dominate over the gluon density. Here, we find it reasonable to rely upon collinear Dokshitzer-Gribov-LipatovAltarelli-Parisi (DGLAP) factorization scheme [27], which provides better theoretical grounds in the large- $x$ region. So, we consider a combination of two techniques, with each of them being used at the kinematic conditions in which it is best suitable [gluon induced subprocess (1) at small $x$ and quark-induced subprocesses (3)-(6) at large $x$ values]. Such a combined scheme was successfully applied to describe the associated production of prompt photons (or $Z$ bosons) and heavy quark jets at the LHC [16,17]. The calculation of production amplitudes (3)-(6) is very straightforward, and a cross-check of our results has been done using the MADGRAPH tool [28].

Note that numerically we keep only valence quarks in (3) to avoid any double-counting. The calculations based on another TMD scenario, the parton branching (PB) approach [29,30], should include both sea and valence quark contributions. Similarly to conventional DGLAPbased evaluations, in order to describe the data, the PB calculations have to involve a number of additional higher-order subprocesses (such as the $q g \rightarrow \gamma q g$ subprocess) properly matched with leading-order terms (see, e.g., Ref. [31]). The relation between the PB and the CCFM scheme applied here (see below) is out of our present consideration.

It is well known that photons may also originate from the so-called fragmentation processes of partons produced in the hard interaction. However, an isolation requirement which is applied in the measurements [1-6] significantly reduces the rate for these processes: after applying the isolation cuts, such contributions amount only to about $10 \%$ of the visible cross section. Therefore, below, we will neglect the contributions from the fragmentation mechanisms. $^{2}$

As usual, to calculate the contributions of quark-induced subprocesses (3)-(6), one has to convolute the corresponding partonic cross sections $d \hat{\sigma}_{a b}$ with the conventional parton distribution functions $f_{a}\left(x, \mu^{2}\right)$ in a proton,

$\sigma=\int d x_{1} d x_{2} d \hat{\sigma}_{a b}\left(x_{1}, x_{2}, \mu^{2}\right) f_{a}\left(x_{1}, \mu^{2}\right) f_{b}\left(x_{2}, \mu^{2}\right)$,

where indices $a$ and $b$ denote quark and/or gluon and $x_{1}$ and $x_{2}$ are the longitudinal momentum fractions of the colliding protons. In the case of off-shell gluon-gluon fusion (1), we employ the $k_{T}$-factorization formula

\footnotetext{
${ }^{2}$ The isolation requirement and additional conditions which preserve our calculations from divergences have been specially discussed in Ref. [24].
} 


$$
\sigma=\int d x_{1} d x_{2} d \mathbf{k}_{1 T}^{2} d \mathbf{k}_{2 T}^{2} d \hat{\sigma}_{g g}^{*}\left(x_{1}, x_{2}, \mathbf{k}_{1 T}^{2}, \mathbf{k}_{2 T}^{2}, \mu^{2}\right) f_{g}\left(x_{1}, \mathbf{k}_{1 T}^{2}, \mu^{2}\right) f_{g}\left(x_{2}, \mathbf{k}_{2 T}^{2}, \mu^{2}\right),
$$

where $f_{g}\left(x, \mathbf{k}_{T}^{2}, \mu^{2}\right)$ is the TMD gluon density in a proton. A comprehensive collection of the latter can be found in the TMDLIB package [32], which is a C++ library providing a framework and an interface to the different parametrizations. In the present paper, we have tested the two latest sets (namely, JH'2013 set 1 and JH'2013 set 2), which were obtained [33] from the numerical solution of the CCFM gluon evolution equation. These TMD gluon distribitions are commonly used in the phenomenological applications and were successfully applied recently, for example, for inclusive Higgs boson production [34,35], associated production of prompt photons or gauge bosons and heavy quarks $[16,17,36]$, and prompt and nonprompt charmonia production [37-40] at the LHC. In our opinion, the CCFM equation provides a suitable tool since it smoothly interpolates between the small- $x$ BFKL gluon dynamics and the high- $x$ DGLAP one. The input parameters of the initial gluon distribution were fitted from the best description of the precision deep inelastic scattering data on the proton structure functions $F_{2}\left(x, Q^{2}\right)$ and $F_{2}^{c}\left(x, Q^{2}\right)$. Another popular set of TMD parton distributions, based on the Kimber-Martin-Ryskin approach [41], is out of our present consideration since it basically relies on the DGLAP evolution with transverse momentum introduced at the last evolution step, so the TMD parton showering would not be really effective in this case. ${ }^{3}$ For the conventional quark and gluon densities, we used the MSTW2008 (LO) set [43]. Numerical calculations at the parton level in the $k_{T^{-}}$ factorization approach and collinear QCD factorization were performed using the Monte Carlo event generator PEGASUS.

A last important point of our calculations is connected with the proper determination of the associated jet 4momentum: the quarks and gluons produced in the hard subprocesses (1), (3)-(6) can form final-state hadronic jets. In addition to that, the produced photon is accompanied by a number of gluons radiated in the course of the noncollinear evolution, which also give rise to final jets. From all of these hadronic jets, we choose the one (i.e., leading jet) carrying the largest transverse momentum (and satisfying the experimental cuts) and then compute the cross section of $\gamma+$ jet production. Technically, we produce a Les Houches event file [44] in our parton level calculations performed using the Monte-Carlo event generator PEGASUS and then process the file with a TMD shower implemented in CASCADE, thus fully reconstructing the CCFM evolution. This approach gives us the possibility of taking into

\footnotetext{
${ }^{3}$ The application of the newly developed PB set [42] is briefly discussed in Sec. III.
}

account the contributions from initial-state parton showers in a consistent way and, of course, essentially differs from the simple model [20] used in the previous calculations [19]. This model [20] was based on the assumption that the gluon, emitted in the last noncollinear evolution step, compensates the whole transverse momentum of the gluon participating in the hard subprocess. Under this assumption, all the other emitted gluons can be collected together in the proton remnant, which carries only a negligible transverse momentum (see Ref. [20] for more information). Thus, in this simple approach, we have only two partonic jets in addition to that produced in a hard subprocess, from which we choose the leading jet. Moreover, since the rapidities of both these jets are not predicted by the model [20], one has to take them to be uniformly distributed, which, of course, spoils the momentum conversation and leads, in particular, to an unsatisfactory description of angular correlations between the produced photon and jet at the LHC [19] and $\eta^{\text {jet }}$ and $x^{\gamma}$ distributions at HERA [45]. These disadvantages of the model [20] are absent in the consistent TMD parton shower approach as implemented in CASCADE.

Concerning the quark-induced subprocesses (3)-(6), calculated in the conventional (collinear) QCD factorization, we used the latest version of the PYTHIA package [46] to process the Les Houches event file generated by PEGASUS. The jets are reconstructed with the anti- $k_{T}$ algorithm, implemented in the FastJet tool [47].

\section{NUMERICAL RESULTS}

Throughout this paper, all calculations are based on the following parameter setting. In collinear QCD factorization, we use one-loop strong coupling with $n_{f}=4$ massless quark flavors and $\Lambda_{\mathrm{QCD}}=200 \mathrm{MeV}$, and the factorization and renormalization scales are both set equal to the produced photon transverse energy, $\mu_{R}=\mu_{F}=E_{T}^{\gamma}$.

TABLE I. The kinematical cuts and anti- $k_{T}$ algorithm radius $R^{\text {jet }}$ implemented in the experimental analyses $[1,3,4]$ and in our calculations.

\begin{tabular}{lccc}
\hline \hline & CMS [1] & ATLAS [3] & ATLAS [4] \\
\hline$E_{T}^{\gamma} / \mathrm{GeV}$ & $>40$ & $>45$ & $>25$ \\
$p_{T}^{\text {jet }} / \mathrm{GeV}$ & $>30$ & $>40$ & $>20$ \\
$\left|\eta^{\gamma}\right|$ & $<2.5$ & $<2.37\left(\right.$ excl. $\left.1.37<\left|\eta^{\gamma}\right|<1.52\right)$ & $<1.37$ \\
$\left|\eta^{\text {jet }}\right|$ & $<2.5$ & $<2.37$ & $<4.4$ \\
$\Delta R^{\gamma-\text { jet }}$ & 0.5 & 1.0 & 1.0 \\
$R^{\text {jet }}$ & 0.5 & 0.6 & 0.4 \\
\hline \hline
\end{tabular}


In the $k_{T}$-factorization calculations, we use a two-loop expression for the strong coupling (as it was originally done in the fit [33]), set $\mu_{R}=E_{T}^{\gamma}$, and define the factorization scale as $\mu_{F}^{2}=\hat{s}+\mathbf{Q}_{T}^{2}$ with $\hat{s}$ and $\mathbf{Q}_{T}$ being the subprocess invariant energy and the net transverse momentum of the initial off-shell gluon pair, respectively. Note that the definition of $\mu_{F}$ is dictated by the CCFM evolution algorithm [33].
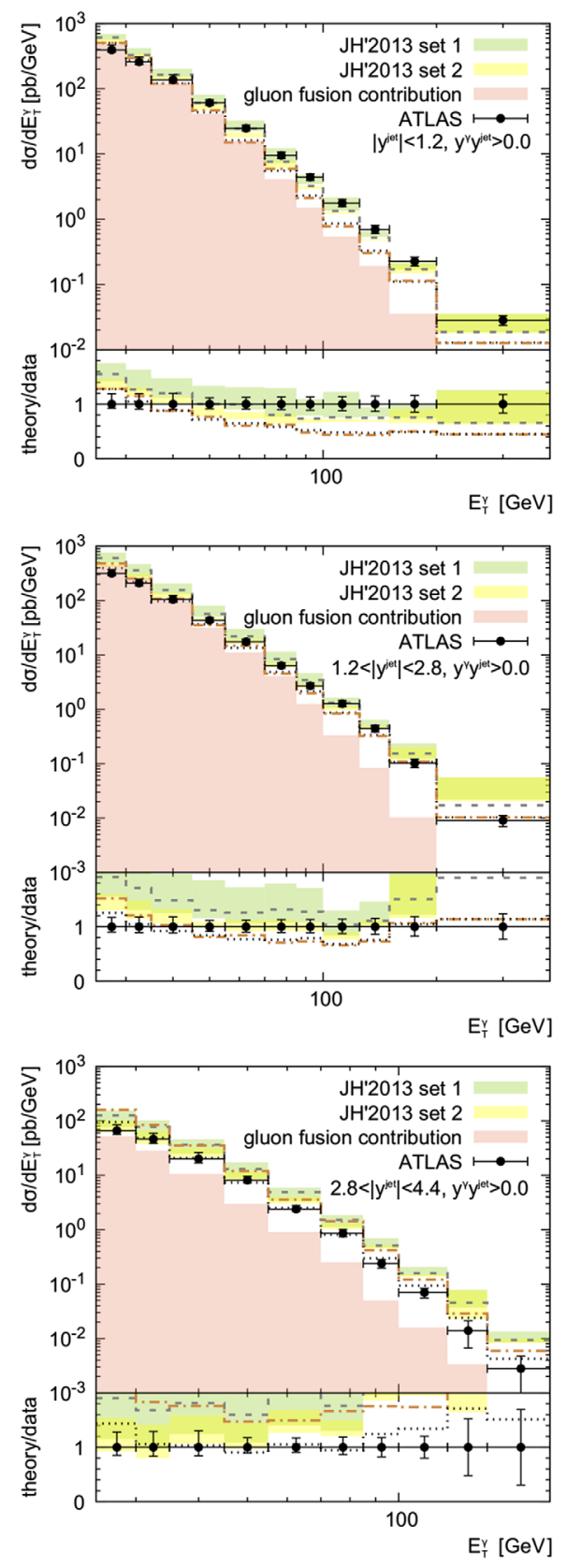

The measurements of associated $\gamma+$ jet production cross sections have been carried out by the CMS [1,2] and ATLAS [3-6] Collaborations at LHC energies $\sqrt{s}=7,8$, and $13 \mathrm{TeV}$. However, the data $[2,5,6]$ refer to the region of high $E_{T}^{\gamma}$ (i.e., the region of relatively large $x \sim E_{T}^{\gamma} / \sqrt{s}$ ), where standard quark-induced subprocesses (3)-(6) dominate. We do not analyze events of this kind in the present study and only concentrate on the small and moderate $E_{T}^{\gamma}$
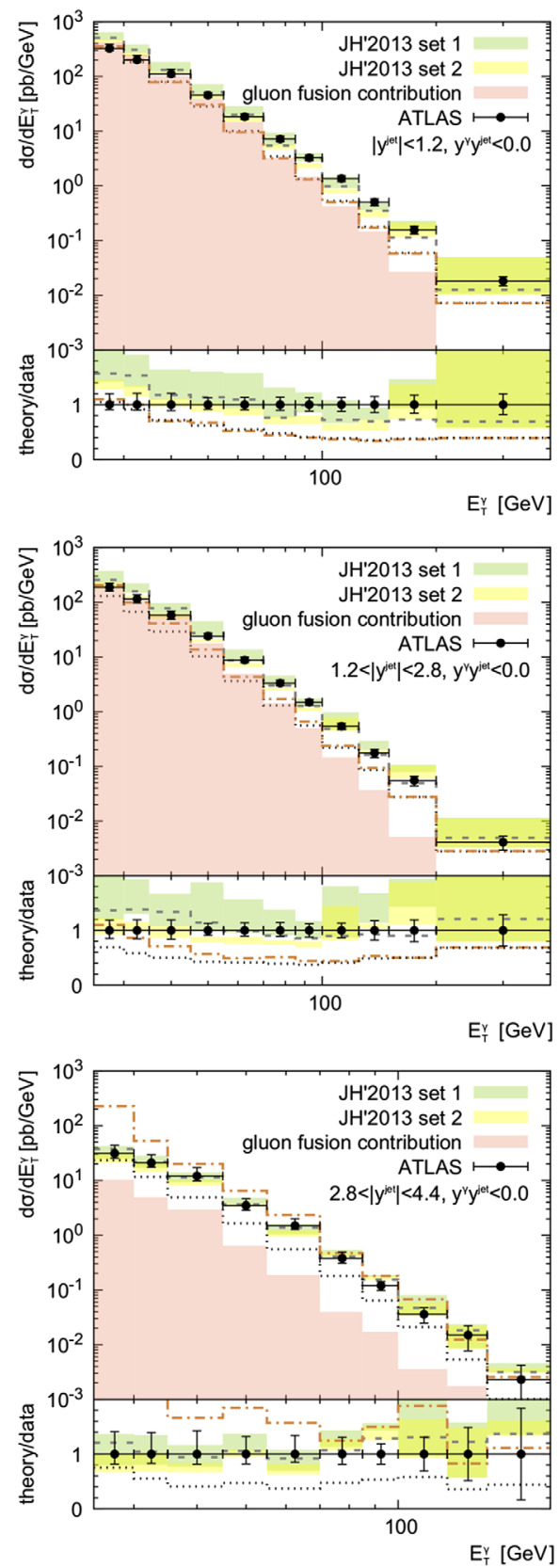

FIG. 1. The differential cross sections of associated $\gamma+$ jet production at $\sqrt{s}=7 \mathrm{TeV}$ as function of the prompt photon transverse energy $E_{T}^{\gamma}$ in different regions of rapidities. The green and yellow shaded bands represent the results obtained with JH'2013 set 1 and set 2 gluon densities (with scale uncertainties). Dashed histograms corresponds to the predictions with initial-state parton showers, dotted histograms represent the results obtained without parton showers, and dashed-dotted histograms correspond to the results, obtained with the simple approach [20]. Contributions from the off-shell gluon fusion subprocess (1) are shown separately. Everywhere, the JH'2013 set 1 gluon density was used. The experimental data are from ATLAS [3]. 

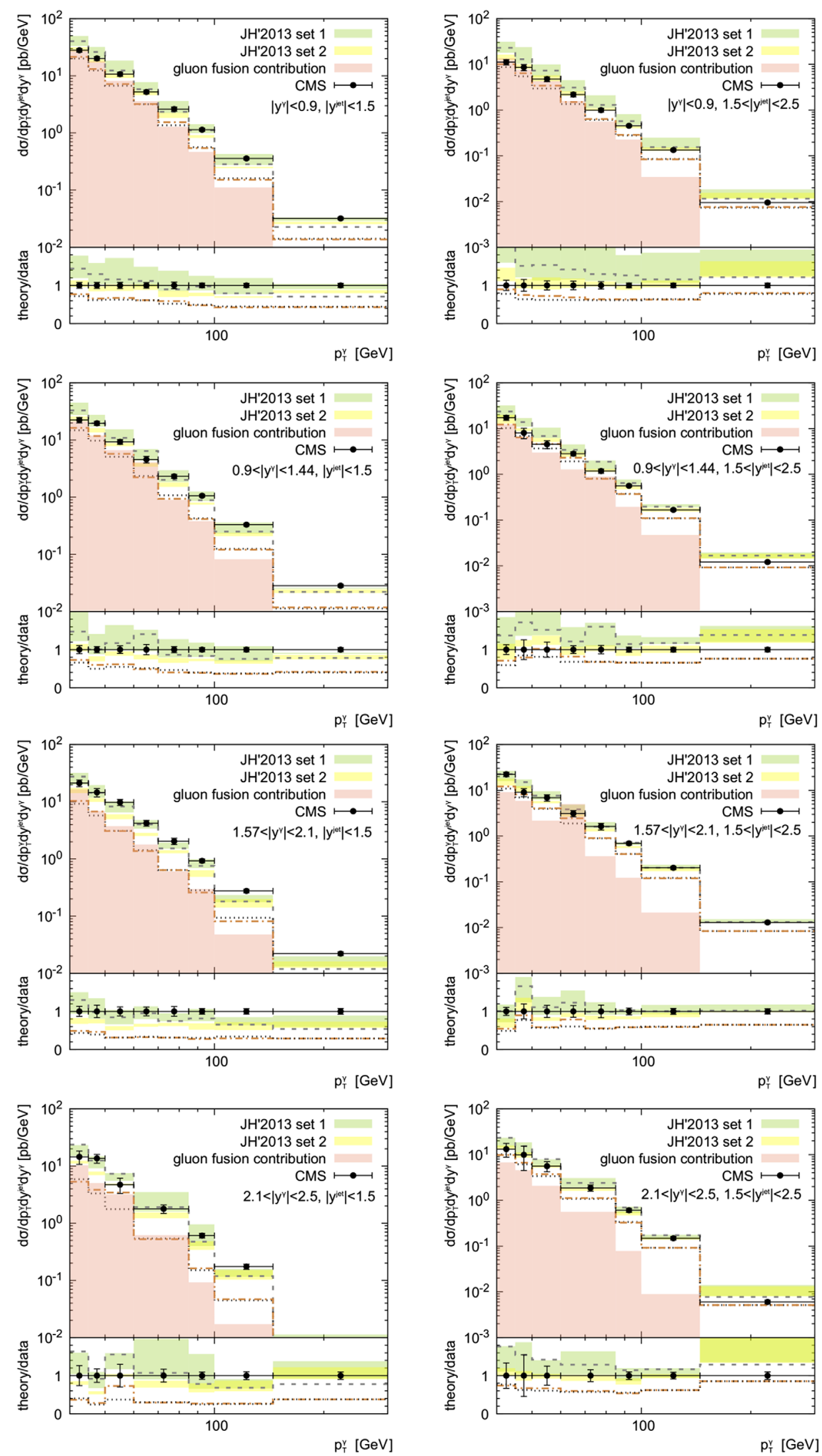

FIG. 2. The triple-differential cross sections of associated $\gamma+$ jet production at $\sqrt{s}=7 \mathrm{TeV}$ as a function of the photon transverse energy in different regions of rapidities. The notation of histograms is the same as in Fig. 1. The experimental data are from CMS [1]. 
data $[1,3,4]$, in which off-shell gluon-gluon fusion plays a role (see the discussion below). The experimental acceptance, anti- $k_{T}$ algorithm radius $R^{\text {jet }}$, and $\eta-\phi$ separation $\Delta R^{\gamma-\text { jet }}$ implemented in the experimental analyses [1,3,4] are collected in Table I. The CMS Collaboration has reported [1] the measurements of triple-differential cross section $d \sigma / d E_{T}^{\gamma} d \eta^{\gamma} d \eta^{\text {jet }}$ for various configurations of the photon and leading jet at $\sqrt{s}=7 \mathrm{TeV}$. In the ATLAS analysis [3], the differential cross section $d \sigma / d E_{T}^{\gamma}$ has been measured for three different rapidity ranges of the leading jet: $\left|y^{\text {jet }}\right|<1.2,1.2<\left|y^{\text {jet }}\right|<2.8$ and $2.8<\left|y^{\text {jet }}\right|<4.4$. For each rapidity configuration, the same-sign $\left(\eta^{\gamma} \eta^{\text {jet }}>0\right)$ and opposite-sign $\left(\eta^{\gamma} \eta^{\text {jet }}<0\right)$ cases are studied separately. More recently, the ATLAS Collaboration has presented measurements [4] of $\gamma+$ jet cross sections as a function of the photon transverse energy $E_{T}^{\gamma}$, leading jet transverse momentum $p_{T}^{\text {jet }}$, and rapidity $y^{\text {jet }}$ at the same energy $\sqrt{s}$. In addition, the cross sections as a function of the difference between the azimuthal angles of the photon and jet $\Delta \phi^{\gamma-\text { jet }}$, invariant mass $m^{\gamma-\text { jet}}$, and scattering angle $\cos \theta=$ $\tanh \left(y^{\gamma}-y^{\text {jet }}\right) / 2$ have been reported.

We confront our predictions with the CMS [1] and ATLAS $[3,4]$ data in Figs. 1-4. The results obtained using the JH'2013 set 1 and set 2 gluon densities (including the
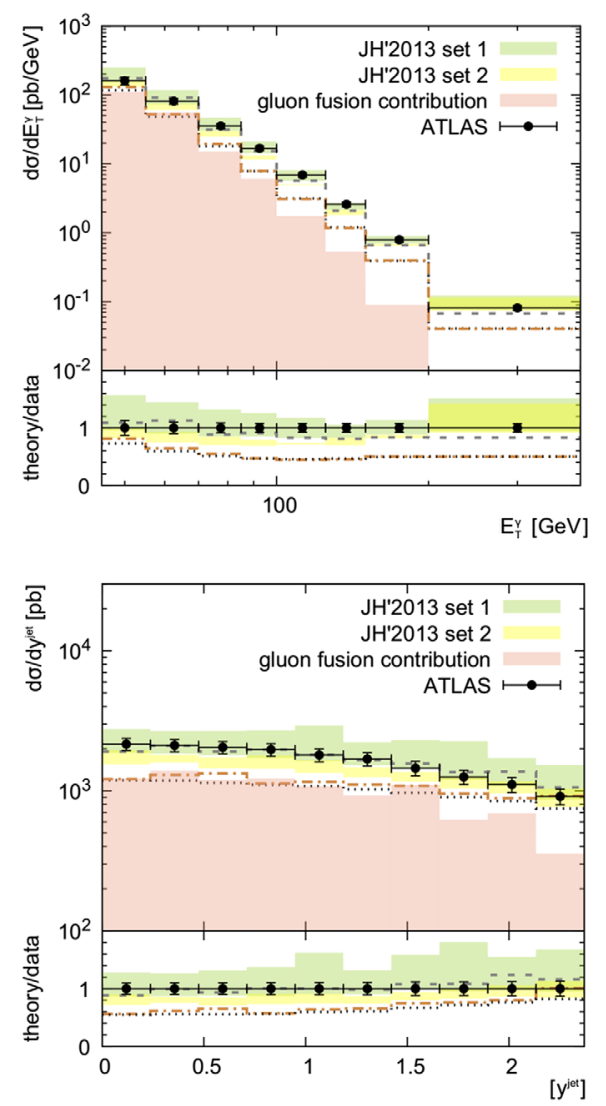

effects of both initial- and final-state parton showers) are plotted with scale uncertainties depicted as green and yellow shaded bands, respectively. To estimate these uncertainties, we used the JH'2013 set 1(2)+ and JH'2013 set 1(2) - gluon distributions instead of default JH'2013 set 1(2) density. These two sets represent a variation of the renormalization scale used in the off-shell production amplitude. The JH'2013 set 1(2)+ stands for a variation of $2 \mu_{R}$, while JH'2013 set $1(2)-$ reflects $\mu_{R} / 2$ (see Ref. [33]). To estimate the scale uncertainties in the quark-involving subprocesses (3)-(6), calculated in the collinear QCD factorization, we have varied the scales $\mu_{R}$ and $\mu_{F}$ by a factor of 2 around their default values. Separately, we show the contribution of offshell gluon-gluon fusion subprocess (1), calculated with $k_{T}$ factorization.

As one can see, we achieved good agreement of our predictions with the CMS [1] and ATLAS $[3,4]$ data in the whole kinematical region within the experimental and theoretical uncertainties. The predictions from the JH'2013 set 2 gluon are somewhat lower than those from JH'2013 set 1, especially for the distribution in scattering angle $\theta$ (see Fig. 4). The reason for this lies in the additional limitation of the phase space in these
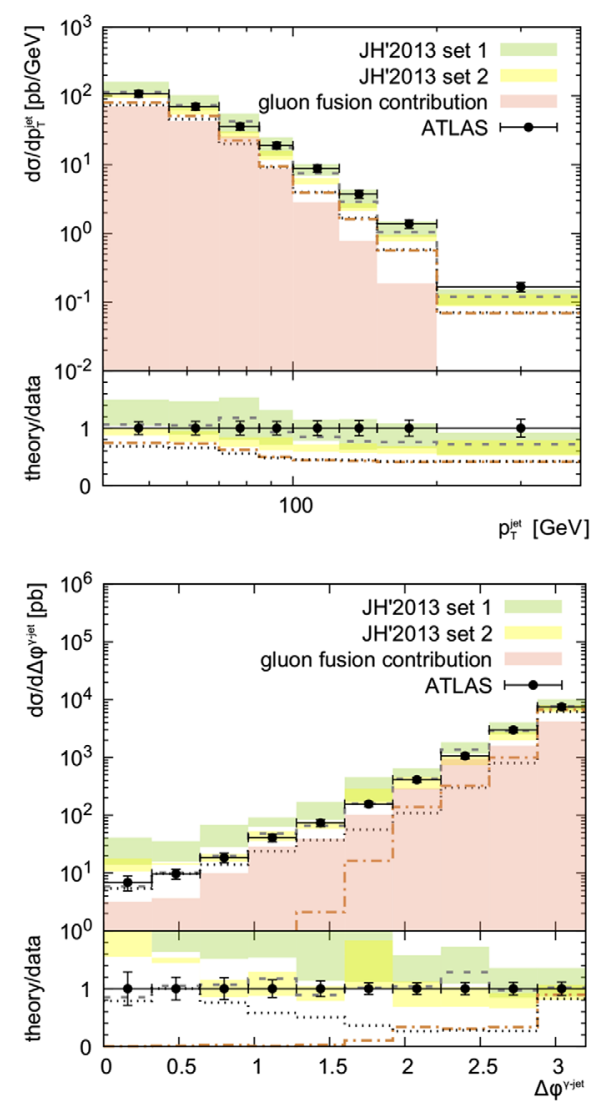

FIG. 3. The differential cross sections of associated prompt photon and jet production at $\sqrt{s}=7 \mathrm{TeV}$ as functions of photon transverse energy $E_{T}^{\gamma}$, jet transverse momentum $p_{T}^{\text {jet }}$, jet rapidity $y^{\text {jet }}$, and the azimuthal angle difference between the prompt photon and the leading jet $\Delta \phi$. The notation of histograms is the same as in Fig. 1. The experimental data are from ATLAS [4]. 

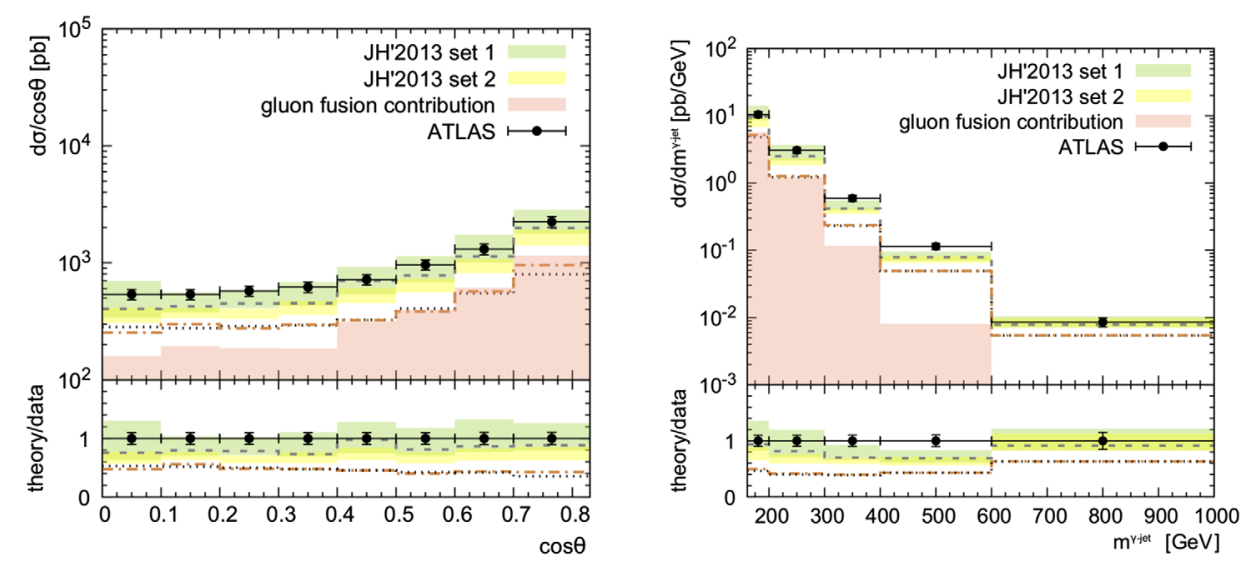

FIG. 4. The differential cross sections of associated prompt photon and jet production at $\sqrt{s}=7$ TeV as function of scattering angle $\cos \theta$ and the invariant mass of the prompt photon and the leading jet. Additional cuts $\cos \theta<0.83, m^{\gamma-\text { jet }}>161 \mathrm{GeV}$ and $\left|y^{\gamma}+y^{\text {jet }}\right|<$ 2.37 are applied. The notation of histograms is the same as in Fig. 1. The experimental data are from ATLAS [4].

measurements [4], namely, $\cos \theta<0.83, \quad m^{\gamma-\text { jet }}>$ $161 \mathrm{GeV}$ and $\left|y^{\gamma}+y^{\mathrm{jet}}\right|<2.37$, which moves the probed kinematical region to somewhat larger $x$. Note that the measured distribution in $\cos \theta$ is sensitive to the $\gamma+$ jet production dynamics and well reproduced in our calculations with the JH'2013 set 1 gluon. One can see also that the off-shell gluon-gluon fusion subprocess (1), in which we are mainly interested, dominates at low and moderate transverse energy $\left(E_{T}^{\gamma} \leq 120\right.$ or $\left.150 \mathrm{GeV}\right)$ and practically does not contribute at larger values. ${ }^{4}$ So, the subleading quark-induced subprocesses (3)-(6) are important to achieve an adequate description of the data in the whole $E_{T}^{\gamma}$ region. Similar conclusions were made earlier in Ref. [17] in the case of associated $Z$ boson and heavy quark jet production at the LHC.

As was noted above, the initial-state parton shower in CASCADE is based on the CCFM evolution equation, while the final state parton shower is based on the DGLAP equations. To investigate the influence of parton showers in a final state for the description of the LHC data, we repeated the calculations, taking into account parton showers in the initial state only. These results are presented in Figs. 1-4 by dashed histograms. We find that the final-state radiation effects are quite negligible in most of the distributions, excluding only the region of very small $\Delta \phi^{\gamma-\text { jet }}$ (see Fig. 3). In addition, we show the predictions obtained with no parton showers in initial state (the dotted histograms in Figs. 1-4). These results

\footnotetext{
${ }^{4}$ We have tested also newly proposed TMD gluon densities [42] obtained as solutions of DGLAP equations with keeping exact kinematics using the PB method $[29,30]$. The PB-based predictions (not shown in Figs. 1-4) significantly underestimate the data, which demonstrates the role of small- $x$ resummation in the CCFM equation. The necessity of taking into account of higher-order QCD corrections when using the PB parton densities has been demonstrated recently in Ref. [48].
}

clearly illustrate the importance of the latter for description of the data.

For a last point of our study, we present results of our calculations in which the simple model [20] has been applied in the jet selection procedure, similar to previous evaluations [19] (dashed-dotted histograms in Figs. 1-4). As one can see, the achieved overall description of the considered experimental data is systematically worse, both in normalization and shape. Although the simple approach [20] is able to describe more or less adequately the measured $E_{T}^{\gamma}$ distributions in the some kinematical region (as is shown in Fig. 2), it fails for more exclusive observables, such as the $\Delta \phi^{\gamma-\text { jet }}$ variable (see Fig. 3). Thus, it indicates again the importance of taking into account contributions from initial-state parton showers for the proper determination of the leading jet in the $k_{T^{-}}$ factorization approach.

\section{CONCLUSION}

We have considered associated production of prompt photon and hadronic jets at LHC conditions. The calculations were performed in a "combined" scheme, employing both $k_{T}$ factorization and collinear factorization in QCD, with each of them used in the kinematic conditions of its best reliability. The dominant contribution is represented by the off-shell gluon-gluon fusion subprocess $g^{*} g^{*} \rightarrow \gamma q \bar{q}$. Several subleading quark-induced subprocesses contributing at $\mathcal{O}\left(\alpha \alpha_{s}\right)$ and $\mathcal{O}\left(\alpha \alpha_{s}^{2}\right)$ have been taken into account in the conventional collinear scheme. To reconstruct correctly the kinematics of the hadronic jets, the TMD parton shower generator CASCADE has been applied.

Using the TMD gluon densities derived from the CCFM evolution equation, we have achieved reasonably good agreement between our theoretical predictions and the CMS and ATLAS experimental data. We have 
demonstrated the importance of initial-state parton showers for jet determination in the $k_{T}$-factorization approach.

\section{ACKNOWLEDGMENTS}

The authors thank S.P. Baranov for very useful discussions and important remarks. A. V. L. and M. A. M. are grateful to DESY Directorate for the support in the framework of Cooperation Agreement between MSU and DESY on phenomenology of the LHC processes and TMD parton densities. M. A. M. was also supported by a grant of the foundation for the advancement of theoretical physics and mathematics "Basis," Grant No. 17-14-455-1.
[1] CMS Collaboration, J. High Energy Phys. 06 (2014) 009.

[2] CMS Collaboration, Eur. Phys. J. C 79, 20 (2019).

[3] ATLAS Collaboration, Phys. Rev. D 85, 092014 (2012).

[4] ATLAS Collaboration, Nucl. Phys. B875, 483 (2013).

[5] ATLAS Collaboration, Nucl. Phys. B918, 257 (2017).

[6] ATLAS Collaboration, Phys. Lett. B 780, 578 (2018).

[7] S. Catani, M. Fontannaz, J.-Ph. Guillet, and E. Pilon, J. High Energy Phys. 05 (2002) 028; P. Aurenche, M. Fontannaz, J.-Ph. Guillet, E. Pilon, and M. Werlen, Phys. Rev. D 73, 094007 (2006); Z. Belghobsi, M. Fontannaz, J.-Ph. Guillet, G. Heinrich, E. Pilon, and M. Werlen, Phys. Rev. D 79, 114024 (2009).

[8] T. Gleisberg, S. Hoeche, F. Krauss, M. Schoenherr, S. Schumann, F. Siegert, and J. Winter, J. High Energy Phys. 02 (2009) 007.

[9] S. Catani, M. Ciafaloni, and F. Hautmann, Nucl. Phys. B366, 135 (1991); J. C. Collins and R. K. Ellis, Nucl. Phys. B360, 3 (1991).

[10] L. V. Gribov, E. M. Levin, and M. G. Ryskin, Phys. Rep. 100, 1 (1983); E. M. Levin, M. G. Ryskin, Yu. M. Shabelsky, and A. G. Shuvaev, Sov. J. Nucl. Phys. 53, 657 (1991).

[11] E. A. Kuraev, L. N. Lipatov, and V. S. Fadin, Sov. Phys. JETP 44, 443 (1976); 45, 199 (1977); I. I. Balitsky and L. N. Lipatov, Sov. J. Nucl. Phys. 28, 822 (1978).

[12] M. Ciafaloni, Nucl. Phys. B296, 49 (1988); S. Catani, F. Fiorani, and G. Marchesini, Phys. Lett. B 234, 339 (1990); Nucl. Phys. B336, 18 (1990); G. Marchesini, Nucl. Phys. B445, 49 (1995).

[13] R. Angeles-Martinez et al., Acta Phys. Pol. B 46, 2501 (2015).

[14] B. Andersson et al. (Small-x Collaboration), Eur. Phys. J. C 25, 77 (2002); J. Andersen et al. (Small-x Collaboration), Eur. Phys. J. C 35, 67 (2004); 48, 53 (2006).

[15] A. V. Lipatov and M. A. Malyshev, Phys. Rev. D 94, 034020 (2016)

[16] V. A. Bednyakov, S. J. Brodsky, A. V. Lipatov, G. I. Lykasov, M. A. Malyshev, J. Smiesko, and S. Tokar, Eur. Phys. J. C 79, 92 (2019).

[17] S. P. Baranov, H. Jung, A. V. Lipatov, and M. A. Malyshev, Eur. Phys. J. C 77, 772 (2017).

[18] T. Pietrycki and A. Szczurek, Phys. Rev. D 76, 034003 (2007).

[19] A. V. Lipatov and N. P. Zotov, Phys. Rev. D 90, 094005 (2014).
[20] S. P. Baranov and N. P. Zotov, Phys. Lett. B 491, 111 (2000).

[21] M. Deak, F. Hautmann, H. Jung, and K. Kutak, arXiv:1206.7090.

[22] S. Dooling, F. Hautmann, and H. Jung, Phys. Lett. B 736, 293 (2014).

[23] H. Jung, S. P. Baranov, M. Deak, A. Grebenyuk, F. Hautmann, M. Hentschinski, A. Knutsson, M. Kramer, K. Kutak, A. V. Lipatov, and N. P. Zotov, Eur. Phys. J. C 70, 1237 (2010).

[24] S. P. Baranov, A. V. Lipatov, and N. P. Zotov, Phys. Rev. D 78, 014025 (2008).

[25] M. Deak and F. Schwennsen, J. High Energy Phys. 09 (2008) 035.

[26] S. P. Baranov, A. V. Lipatov, and M. A. Malyshev (to be published).

[27] V. N. Gribov and L. N. Lipatov, Sov. J. Nucl. Phys. 15, 438 (1972); L. N. Lipatov, Sov. J. Nucl. Phys. 20, 94 (1975); G. Altarelli and G. Parisi, Nucl. Phys. B126, 298 (1977); Yu. L. Dokshitzer, Sov. Phys. JETP 46, 641 (1977).

[28] J. Alwall, R. Frederix, S. Frixione, V. Hirschi, F. Maltoni, O. Mattelaer, H.-S. Shao, T. Stelzer, P. Torrielli, and M. Zaro, J. High Energy Phys. 07 (2014) 079.

[29] F. Hautmann, H. Jung, A. Lelek, V. Radescu, and R. Zlebcik, Phys. Lett. B 772, 446 (2017).

[30] F. Hautmann, H. Jung, A. Lelek, V. Radescu, and R. Zlebcik, J. High Energy Phys. 01 (2018) 070.

[31] A. B. Martinez, P. Connor, D. D. Damiani, L. I. E. Banos, F. Hautmann, H. Jung, J. Lidrych, M. Schmitz, S. T. Monfared, Q. Wang, and R. Zlebcik, arXiv:1906.00919.

[32] http://tmd.hepforge.org.

[33] F. Hautmann and H. Jung, Nucl. Phys. B883, 1 (2014).

[34] N. A. Abdulov, A. V. Lipatov, and M. A. Malyshev, Phys. Rev. D 97, 054017 (2018).

[35] R. Islam, M. Kumar, and V. S. Rawoot, Eur. Phys. J. C 79, 181 (2019).

[36] A. V. Lipatov, G. I. Lykasov, M. A. Malyshev, A. A. Prokhorov, and S. M. Turchikhin, Phys. Rev. D 97, 114019 (2018).

[37] A. V. Lipatov, S. P. Baranov, H. Jung, and M. A. Malyshev, Eur. Phys. J. C 78, 2 (2018).

[38] S. P. Baranov, A. V. Lipatov, and M. A. Malyshev, Eur. Phys. J. C 78, 820 (2018).

[39] S. P. Baranov and A. V. Lipatov, Eur. Phys. J. C 79, 621 (2019). 
[40] S. P. Baranov and A. V. Lipatov, arXiv:1906.07182.

[41] M. A. Kimber, A. D. Martin, and M. G. Ryskin, Phys. Rev. D 63, 114027 (2001); A. D. Martin, M. G. Ryskin, and G. Watt, Eur. Phys. J. C 31, 73 (2003); 66, 163 (2010).

[42] A. B. Martinez, P. Connor, F. Hautmann, H. Jung, A. Lelek, V. Radescu, and R. Zlebcik, Phys. Rev. D 99, 074008 (2019).

[43] A. D. Martin, W. J. Stirling, R. S. Thorne, and G. Watt, Eur. Phys. J. C 63, 189 (2009).
[44] J. Alwall et al., Comput. Phys. Commun. 176, 300 (2007).

[45] A. V. Lipatov, M. A. Malyshev, and N. P. Zotov, Phys. Rev. D 88, 074001 (2013).

[46] T. Sjöstrand, S. Ask, J. R. Christiansen, R. Corke, N. Desai, P. Ilten, S. Mrenna, S. Prestel, C. O. Rasmussen, and P. Z. Skands, Comput. Phys. Commun. 191, 159 (2015).

[47] M. Cacciari, G. P. Salam, and G. Soyez, Eur. Phys. J. C 72, 1896 (2012).

[48] R. Maciula and A. Szczurek, arXiv:1905.06697. 